\title{
SOME NEW PDE METHODS FOR WEAK KAM THEORY
}

\author{
LAWRENCE C. Evans ${ }^{1}$
}

\begin{abstract}
We discuss a new approximate variational principle for weak KAM theory. The advantage of this approach is that we build both a minimizing measure and a solution of the generalized eikonal equation at the same time. Furthermore the approximations are smooth, and so we can derive some interesting formulas upon differentiating the Euler-Lagrange equation.

Our method is inspired by the "calculus of variations in the sup-norm" ideas of Aronsson, Jensen, Barron and others.
\end{abstract}

\section{Introduction.}

This paper provides a new variational and PDE approach to Mather's minimization principle in dynamics, set forth in [Mt1-2], and to weak KAM theory, earlier developed in Fathi [F1-3] and in [E-G]. An overall goal of this work is to identify a sort of integrable structure within certain general classes of Hamiltonian dynamics and to understand how dynamical information is encoded in a related "effective Hamiltonian". See Mather-Forni $[\mathrm{M}-\mathrm{F}]$, Fathi [F3] or the introduction to [E-G] for more background.

We are motivated here by the min-max formula

$$
\bar{H}(P)=\inf _{v \in C^{1}\left(\mathbb{T}^{n}\right)} \max _{x \in \mathbb{T}^{n}} H(P+D v, x),
$$

where the Hamiltonian $H$ satisfies some conditions listed below, most notably periodicity in $x$ over the unit flat torus $\mathbb{T}^{n}$. The term $\bar{H}$ denotes the effective Hamiltonian in the sense of Lions-Papanicolaou-Varadhan [L-P-V] (which is equivalent in our setting to Mather's function $\alpha$ ). Several authors, among them Fathi, Mañé, Contreras-Iturriaga-Paternain and Gomes, have independently derived this identity. For the reader's convenience, we reproduce in Appendix B $(\S 6)$ a quick proof that Fathi recently found.

A sup-norm variational principle. Formula (1.1) suggests that we can compute $\bar{H}(P)$ by trying to minimize the sup-norm of $H(P+D v, x)$ over $\mathbb{T}^{n}$. This viewpoint is

${ }^{1}$ Supported in part by NSF Grant DMS-0070480 and by the Miller Institute for Basic Research in Science, UC Berkeley 
strongly reminiscent of "Aronsson's variational principle" in the calculus of variations: See for instance Aronsson [A1-2], Barron [B], Barron-Jensen-Wang [B-J-W], etc. The idea is that we should not just try to minimize the sup-norm of $H(P+D v, x)$ over all of $\mathbb{T}^{n}$, but rather we should look for a function $v$ which minimizes

$$
\|H(P+D v, x)\|_{L^{\infty}(U)}
$$

relative to its boundary values, for each open subdomain $U \subset \mathbb{T}^{n}$. We then call $v$ an absolute minimizer.

The references cited above suggest also that we can search for absolute minimizers by replacing the sup-norm by an $L^{p}$-norm and letting $p \rightarrow \infty$. That is, for each $1 \leq p<\infty$ we should find $v^{p} \in C^{1}\left(\mathbb{T}^{n}\right)$ to minimize the functional

$$
\int_{\mathbb{T}^{n}}\left|H\left(P+D v^{p}, x\right)\right|^{p} d x
$$

and then study the limiting behavior of the functions $v^{p}$ when $p \rightarrow \infty$. (Paternain $[\mathrm{P}]$ discusses a somewhat related variational formulation, but without sending $p \rightarrow \infty$.)

It is however more elegant to employ exponentials rather than powers, and some of the resulting computations resemble the formalism of statistical mechanics. We will therefore for this paper first take a positive integer $k$, and look then for $v^{k} \in C^{1}\left(\mathbb{T}^{n}\right)$ to minimize the functional

$$
I_{k}[v]:=\int_{\mathbb{T}^{n}} e^{k H\left(P+D v^{k}, x\right)} d x
$$

Under the following assumptions on $H$, there exists a minimizer $v^{k} \in C^{\infty}\left(\mathbb{T}^{n}\right)$, which is unique once we require

$$
\int_{\mathbb{T}^{n}} v^{k} d x=0
$$

See Appendix A in $\S 5$ for more details.

Hypotheses on the Hamiltonian: We suppose $H: \mathbb{R}^{n} \times \mathbb{R}^{n} \rightarrow \mathbb{R}, H=H(p, x)$, is smooth and satisfies these conditions:

(i) periodicity in $x$ : For each $p \in \mathbb{R}^{n}$, the mapping $x \mapsto H(p, x)$ is $\mathbb{T}^{n}$-periodic.

(ii) strict convexity: There exists a constant $\gamma>0$ such that

$$
\gamma|\xi|^{2} \leq H_{p_{i} p_{j}}(p, x) \xi_{i} \xi_{j}
$$

for each $p, x, \xi \in \mathbb{R}^{n}$. 
(iii) growth bounds: There exists a constant $C$ such that

$$
\begin{aligned}
& \left|D_{p}^{2} H(p, x)\right| \leq C \\
& \left|D_{x, p}^{2} H(p, x)\right| \leq C(1+|p|) \quad\left(p, x \in \mathbb{R}^{n}\right) . \\
& \left|D_{x}^{2} H(p, x)\right| \leq C\left(1+|p|^{2}\right)
\end{aligned}
$$

These assumptions imply the following growth estimates for $H$ and its first derivatives:

$$
\begin{gathered}
\frac{\gamma}{2}|p|^{2}-C \leq H(p, x) \leq C\left(1+|p|^{2}\right) \quad\left(p, x \in \mathbb{R}^{n}\right), \\
\left|D_{p} H(p, x)\right| \leq C(1+|p|) \\
\left|D_{x} H(p, x)\right| \leq C\left(1+|p|^{2}\right) \quad\left(p, x \in \mathbb{R}^{n}\right) .
\end{gathered}
$$

The corresponding Lagrangian is

$$
L(q, x):=\max _{p}(p \cdot q-H(p, x)) \quad\left(q, x \in \mathbb{R}^{n}\right)
$$

PDE interpretations. The Euler-Lagrange equation for our minimizer of $I_{k}[\cdot]$ is

$$
\operatorname{div}\left(e^{k H\left(D u^{k}, x\right)} D_{p} H\left(D u^{k}, x\right)\right)=0
$$

or, equivalently,

$$
\begin{aligned}
\frac{1}{k}\left(H_{p_{i}}\left(D u^{k}, x\right)\right)_{x_{i}}+ & H_{p_{i}}\left(D u^{k}, x\right) H_{p_{j}}\left(D u^{k}, x\right) u_{x_{i} x_{j}}^{k} \\
& +H_{x_{i}}\left(D u^{k}, x\right) H_{p_{i}}\left(D u^{k}, x\right)=0
\end{aligned}
$$

where we have set

$$
u^{k}:=P \cdot x+v^{k} .
$$

Define also

$$
\sigma^{k}:=\frac{e^{k H\left(D u^{k}, x\right)}}{\int_{\mathbb{T}^{n}} e^{k H\left(D u^{k}, x\right)} d x}=e^{k\left(H\left(D u^{k}, x\right)-\bar{H}^{k}(P)\right)},
$$

for

$$
\bar{H}^{k}(P):=\frac{1}{k} \log \left(\int_{\mathbb{T}^{n}} e^{k H\left(D u^{k}, x\right)} d x\right) .
$$

Then

$$
\sigma^{k} \geq 0, \quad \int_{\frac{\mathbb{T}^{n}}{3}} d \sigma^{k}=1
$$


where $d \sigma^{k}:=\sigma^{k} d x$. Observe that the Euler-Lagrange equation (1.7) now reads

$$
\operatorname{div}\left(\sigma^{k} D_{p} H\left(D u^{k}, x\right)\right)=0
$$

Overview. In the following sections we attempt to understand the limiting behavior of $u^{k}$ and $\sigma^{k}$ as $k \rightarrow \infty$. In $\S 2$ we extract some convergent subsequences and in part characterize their limits. We then in $\S 3$ lift into phase space, as a technical device to help us refine our understanding of the limit function $u$ and the limit measure $\sigma$. Section 4 provides some formulas for the first and second derivatives of $\bar{H}^{k}$, and as an application discusses consequences of a nonresonance condition.

Appendix A in $\S 5$ records a sup-norm estimate on $\left|D u^{k}\right|$ and discusses the solvability of (1.7). Appendix B in $\S 6$ reproduces Fathi's short proof of min-max formula (1.1).

As we will see, the technique of approximating by the PDE (1.11) is fairly elegant. It is less clear however that this will lead to anything really new, although the structure of (1.11) perhaps suggests that numerical methods for nonlinear elliptic PDE may prove useful for computing $\bar{H}$. There are several papers in the PDE literature that study variational problems with exponential growth nonlinearities. These include Duc-Eells [D-E], Lieberman [L] and Naito [N] (which handles $\mathbb{R}^{m}$-valued mappings). I thank M. Giaquinta and G. Lieberman for these references.

\section{Convergence, approximating the effective Hamiltonian.}

We propose to let $k \rightarrow \infty$, and will need some simple estimates. We may hereafter suppose without loss of generality that

$$
H \geq 0
$$

Lemma 2.1. For each $1 \leq q<\infty$, there exists a constant $C=C(P, q)$ such that

$$
\sup _{k}\left\|u^{k}\right\|_{W^{1, q}\left(\mathbb{T}^{n}\right)} \leq C
$$

Proof. Since $v^{k}$ is a minimizer of $I_{k}[\cdot]$, we can compare $I_{k}\left[v^{k}\right]$ with $I_{k}[0]$, to estimate

$$
\int_{\mathbb{T}^{n}} e^{k H\left(P+D v^{k}, x\right)} d x \leq \int_{\mathbb{T}^{n}} e^{k H(P, x)} d x \leq e^{k C} .
$$

Hence

$$
\left\|e^{H\left(D u^{k}, x\right)}\right\|_{L^{1}\left(\mathbb{T}^{n}\right)} \leq\left\|e^{H\left(D u^{k}, x\right)}\right\|_{L^{k}\left(\mathbb{T}^{n}\right)} \leq e^{C}
$$


and, since $H$ grows quadratically in variable $p$, this bound implies for each $1 \leq q<\infty$ that

$$
\left\|D u^{k}\right\|_{L^{q}\left(\mathbb{T}^{n}\right)} \leq C
$$

Since the integral of $v^{k}$ is zero, we can then estimate the $L^{q}$-norm of $v^{k}$, and so to finish the proof.

Utilizing this Lemma and if necessary passing to a subsequence, we have

$$
u^{k} \rightarrow u=P \cdot x+v \quad \text { uniformly on } \mathbb{T}^{n},
$$

and for each $1 \leq q<\infty$,

$$
D u^{k} \rightarrow D u=P+D v \quad \text { weakly in } L^{q}\left(\mathbb{T}^{n} ; \mathbb{R}^{n}\right),
$$

where $v \in W^{1, q}\left(\mathbb{T}^{n}\right)$.

In addition, in view of (1.10) we may also suppose that

$$
\sigma^{k} \rightarrow \sigma \quad \text { weakly as measures on } \mathbb{T}^{n}
$$

where $\sigma$ is a Radon probability measure on $\mathbb{T}^{n}$.

Theorem 2.1. (i) We have

$$
\bar{H}(P)=\lim _{k \rightarrow \infty} \bar{H}^{k}(P)=\lim _{k \rightarrow \infty} \frac{1}{k} \log \left(\int_{\mathbb{T}^{n}} e^{k H\left(D u^{k}, x\right)} d x\right) .
$$

(ii) The function $u$ is a viscosity solution of Aronsson's equation

$$
-H_{p_{i}}(D u, x) H_{p_{j}}(D u, x) u_{x_{i} x_{j}}=H_{x_{i}}(D u, x) H_{p_{i}}(D u, x) .
$$

(iii) Furthermore,

$$
H(D u, x) \leq \bar{H}(P) \quad \text { a.e. in } \mathbb{T}^{n} .
$$

Proof. 1. According to Lions, Papanicolaou, and Varadhan [L-P-V], there exists a periodic, Lipschitz continuous function $v$ solving

$$
H(P+D v, x)=\bar{H}(P)
$$

in the viscosity sense, and thus also almost everywhere. Then

$$
\int_{\mathbb{T}^{n}} e^{k H\left(P+D v^{k}, x\right)} d x \leq \int_{\mathbb{T}^{n}} e^{k H(P+D v, x)} d x=e^{k \bar{H}(P)},
$$


and consequently

$$
\limsup _{k \rightarrow \infty} \frac{1}{k} \log \left(\int_{\mathbb{T}^{n}} e^{k H\left(D u^{k}, x\right)} d x\right) \leq \bar{H}(P) .
$$

Suppose next that

$$
\liminf _{k \rightarrow \infty} \frac{1}{k} \log \left(\int_{\mathbb{T}^{n}} e^{k H\left(D u^{k}, x\right)} d x\right)<\bar{H}(P)-\varepsilon
$$

for some $\varepsilon>0$. Let

$$
\Lambda_{\varepsilon, k}:=\left\{x \in \mathbb{T}^{n} \mid H\left(D u^{k}, x\right)>\bar{H}(P)-\frac{\varepsilon}{2}\right\}
$$

Then for some sequence $k_{j} \rightarrow \infty$, we have

$$
\frac{1}{k_{j}} \log \left|\Lambda_{\varepsilon, k_{j}}\right|+\bar{H}(P)-\frac{\varepsilon}{2} \leq \bar{H}(P)-\varepsilon .
$$

This inequality implies

$$
\left|\Lambda_{\varepsilon, k_{j}}\right| \leq e^{-k_{j} \frac{\varepsilon}{2}}
$$

Hence for each measurable set $A \subset \mathbb{T}^{n}$, with $|A|>0$,

$$
f_{A} H(D u, x) d x \leq \liminf _{k_{j} \rightarrow \infty} f_{A} H\left(D u^{k_{j}}, x\right) d x \leq \bar{H}(P)-\frac{\varepsilon}{2},
$$

the slash through the integral denoting an average, and so

$$
H(D u, x) \leq \bar{H}(P)-\frac{\varepsilon}{2} \quad \text { a.e. }
$$

We now set $u_{\delta}:=\eta_{\delta} * u$, where $\eta_{\delta}$ denotes a standard mollifier with support in the ball $B(0, \delta)$. Then

$$
H\left(D u_{\delta}, x\right) \leq \bar{H}(P)-\frac{\varepsilon}{4} \quad \text { in } \mathbb{T}^{n}
$$

for sufficiently small $\delta>0$. This however contradicts the min-max formula (1.1) since $u_{\delta}=P \cdot x+v_{\delta}$ for some smooth, periodic function $v_{\delta}$. We have proved assertion (i).

2. Let $u-\phi$ have a strict maximum at a point $x_{0} \in \mathbb{T}^{n}$. Then $u^{k}-\phi$ has a maximum at a nearby point $x_{k}$, with $x_{k} \rightarrow x_{0}$ as $k \rightarrow \infty$. Then (1.7) implies

$$
-\operatorname{div}\left(e^{k H\left(D \phi, x_{k}\right)} D_{p} H\left(D \phi, x_{k}\right)\right) \leq 0
$$

that is,

$$
-\left(H_{p_{j}} \phi_{x_{j} x_{i}}+H_{x_{i}}\right) H_{p_{i}} \leq \frac{1}{k}\left(H_{p_{i}}\right)_{x_{i}}
$$


at the point $x_{k}$. Let $k \rightarrow \infty$, to deduce

$$
-H_{p_{i}}\left(D \phi, x_{0}\right) H_{p_{j}}\left(D \phi, x_{0}\right) \phi_{x_{i} x_{j}} \leq H_{x_{i}}\left(D \phi, x_{0}\right) H_{p_{i}}\left(D \phi, x_{0}\right) .
$$

The opposite inequality holds similarly, should $u-\phi$ attain a strict minimum at $x_{0}$. This proves statement (ii).

3. Let $A \subseteq \mathbb{T}^{n}$ be measurable, with $|A|>0$. Then lower semicontinuity and Jensen's inequality imply

$$
\begin{aligned}
\int_{A} H(D u, x) d x & \leq \liminf _{k \rightarrow \infty} f_{A} H\left(D u^{k}, x\right) d x \\
& \leq \limsup _{k \rightarrow \infty} \frac{1}{k} \log \left(f_{A} e^{k H\left(D u^{k}, x\right)} d x\right) \leq \bar{H}(P)
\end{aligned}
$$

and so $H(D u, x) \leq \bar{H}(P)$ almost everywhere in $\mathbb{T}^{n}$. This is (iii).

\section{Minimizing measures.}

To understand more about the structure of the measure $\sigma$, it is convenient to lift into $\mathbb{R}^{n} \times \mathbb{T}^{n}$, as follows. Define

$$
\mu^{k}:=\delta_{\left\{q=D_{p} H\left(D u^{k}, x\right)\right\}} \sigma^{k}
$$

that is,

$$
\int_{\mathbb{R}^{n}} \int_{\mathbb{T}^{n}} \Phi(q, x) d \mu^{k}=\int_{\mathbb{T}^{n}} \Phi\left(D_{p} H\left(D u^{k}, x\right), x\right) d \sigma^{k}
$$

for each $C^{1}$ function $\Phi$.

Lemma 3.1. The family of probability measures $\left\{\mu^{k}\right\}_{k=1}^{\infty}$ on $\mathbb{R}^{n} \times \mathbb{T}^{n}$ is tight.

Proof. 1. To simplify notation, we temporarily drop the various superscripts $k$. Multiply the Euler-Lagrange PDE $\operatorname{div}\left(\sigma D_{p} H\right)=0$ by $\Delta u$ and integrate by parts over $\mathbb{T}^{n}$, to find

$$
\begin{aligned}
0= & \int_{\mathbb{T}^{n}}\left(\sigma H_{p_{i}}\right)_{x_{j}} u_{x_{i} x_{j}} d x \\
& =\int_{\mathbb{T}^{n}} \sigma\left(H_{p_{i} p_{l}} u_{x_{l} x_{j}}+H_{p_{i} x_{j}}\right) u_{x_{i} x_{j}}+\sigma k h_{x_{j}} H_{p_{i}} u_{x_{i} x_{j}} d x
\end{aligned}
$$

for $h:=H(D u, x)$. Note that $h_{x_{j}}=H_{p_{i}} u_{x_{i} x_{j}}+H_{x_{j}}$. We substitute into the last term above, and obtain

$$
\begin{aligned}
\int_{\mathbb{T}^{n}} \sigma H_{p_{i} p_{l}} u_{x_{l} x_{j}} u_{x_{i} x_{j}} & +\sigma k h_{x_{j}} h_{x_{j}} d x \\
& =\int_{\mathbb{T}^{n}}-\sigma H_{p_{i} x_{j}} u_{x_{i} x_{j}}+\sigma k h_{x_{j}} H_{x_{j}} d x .
\end{aligned}
$$


Observe next that $\sigma_{x_{j}}=\sigma k h_{x_{j}}$ in the last expression, and integrate by parts. After some rewriting, we derive the identity

$$
\int_{\mathbb{T}^{n}} H_{p_{i} p_{l}} u_{x_{l} x_{j}} u_{x_{i} x_{j}}+k h_{x_{j}} h_{x_{j}} d \sigma=-\int_{\mathbb{T}^{n}} 2 H_{p_{i} x_{j}} u_{x_{i} x_{j}}+H_{x_{j} x_{j}} d \sigma .
$$

In view of our hypotheses on $H$, this formula implies the estimate

$$
\int_{\mathbb{T}^{n}} k|D h|^{2} d \sigma \leq C+C \int_{\mathbb{T}^{n}}|D u|^{2} d \sigma \leq C+C \int_{\mathbb{T}^{n}} h d \sigma .
$$

2. Then

$$
\int_{\mathbb{T}^{n}}\left|D \sigma^{\frac{1}{2}}\right|^{2}+\left(\sigma^{\frac{1}{2}}\right)^{2} d x=\int_{\mathbb{T}^{n}} \frac{k^{2}}{4}|D h|^{2} d \sigma+1 \leq k C+C \int_{\mathbb{T}^{n}} k h d \sigma,
$$

and so Sobolev's inequality implies

$$
\left(\int_{\mathbb{T}^{n}} \sigma^{\theta} d \sigma\right)^{\frac{1}{1+\theta}} \leq k C+C \int_{\mathbb{T}^{n}} k h d \sigma
$$

for $\theta=\frac{2}{n-2}$ if $n \geq 3, \theta$ any positive number if $n=2$. Define $\gamma:=\frac{\theta}{2(1+\theta)}$. Then

$$
\begin{aligned}
\left(\int_{\mathbb{T}^{n}} \sigma^{\theta} d \sigma\right)^{\frac{1}{1+\theta}} & \leq k C+C \int_{\mathbb{T}^{n}} k(h-\bar{H})_{+} d \sigma \leq k C+C \int_{\mathbb{T}^{n}} \sigma^{\gamma} d \sigma \\
& \leq k C+C\left(\int_{\mathbb{T}^{n}} \sigma^{\theta} d \sigma\right)^{\frac{1}{2(1+\theta)}} .
\end{aligned}
$$

Therefore

$$
\left(\int_{\mathbb{T}^{n}} \sigma^{\theta} d \sigma\right)^{\frac{1}{1+\theta}} \leq C k .
$$

3. Finally, we restore the superscripts $k$ and estimate for a sufficiently large constant $m$ that

$$
\begin{aligned}
\mu^{k}\{(x, q)|| q \mid \geq m\} & \leq \sigma^{k}\left\{x \mid h \geq \bar{H}^{k}+1\right\} \\
& \leq e^{-k \theta} \int_{\mathbb{T}^{n}} \sigma^{k, \theta} d \sigma^{k} \leq C e^{-k \theta} k^{1+\theta} \rightarrow 0
\end{aligned}
$$

as $k \rightarrow \infty$.

Remark. In Appendix A we derive stronger estimates, showing that in fact the gradients $\left\{D u^{k}\right\}_{k=1}^{\infty}$ are bounded in the sup-norm, uniformly in $k$, and hence the measures $\left\{\mu^{k}\right\}_{k=1}^{\infty}$ have uniformly bounded supports.

Passing to a subsequence if necessary, we may suppose that

$$
\mu^{k} \rightarrow \mu \text { weakly as measures, }
$$

and $\mu$ is a probability measure on $\mathbb{R}^{n} \times \mathbb{T}^{n}$, according to the Lemma. Note that $\sigma=\operatorname{proj}_{x} \mu$, the projection of $\mu$ into $\mathbb{T}^{n}$. Define also the vector

$$
Q:=\int_{\mathbb{R}^{n}} \int_{\mathbb{T}^{n}} q d \mu
$$


Theorem 3.1. (i) The measure $\mu$ is flow invariant; that is,

$$
\int_{\mathbb{R}^{n}} \int_{\mathbb{T}^{n}} q \cdot D \phi d \mu=0
$$

for all $\phi \in C^{1}\left(\mathbb{T}^{n}\right)$.

(ii) The limit

$$
\lim _{k \rightarrow \infty} \int_{\mathbb{T}^{n}} H\left(D u^{k}, x\right) d \sigma^{k}=\bar{H}(P)
$$

holds.

(iii) The measure $\mu$ is a Mather minimizing measure, and in particular

$$
\int_{\mathbb{R}^{n}} \int_{\mathbb{T}^{n}} L(q, x) d \mu=\bar{L}(Q), \quad Q \in \partial \bar{H}(P) .
$$

(iv) The function $u$ is differentiable $\sigma$-almost everywhere and

$$
q=D_{p} H(D u, x) \quad \mu \text {-almost everywhere. }
$$

In particular,

$$
Q:=\int_{\mathbb{T}^{n}} D_{p} H(D u, x) d \sigma
$$

(v) We have

$$
H(D u, x)=\bar{H}(P) \quad \sigma \text {-almost everywhere }
$$

and

$$
\operatorname{div}\left(\sigma D_{p} H\right)=0 \quad \text { in } \mathbb{T}^{n}
$$

The PDE (3.10) and (3.11) are, respectively, the generalized eikonal equation and the transport equation, satisfied by $u$ and $\sigma$. Note we are only asserting that the PDE (3.10) holds on the support of $\sigma$. This is the primary difference between our approach and that of Fathi [F1-3], [E-G]. Recall however that Aronsson's equation (2.2) is valid throughout $\mathbb{T}^{n}$, and so it remains an interesting question to understand what, if anything, this PDE has to do with the underlying Hamiltonian dynamics.

Proof. 1. Let $\phi \in C^{1}\left(\mathbb{T}^{n}\right)$. Then

$$
\int_{\mathbb{R}^{n}} \int_{\mathbb{T}^{n}} q \cdot D \phi d \mu=\lim _{k \rightarrow \infty} \int_{\mathbb{T}^{n}} D_{p} H\left(D u^{k}, x\right) \cdot D \phi d \sigma^{k}=0
$$


since $\operatorname{div}\left(\sigma^{k} D_{p} H\right)=0$ according to (1.11). This proves (3.6).

2. As a first step towards establishing (3.7), let us show that

$$
\bar{H}(P) \leq \liminf _{k \rightarrow \infty} \int_{\mathbb{T}^{n}} H\left(D u^{k}, x\right) d \sigma^{k}
$$

To confirm this, we compute for each $\lambda>0$ that

$$
\begin{aligned}
\bar{H}^{k}(P) & =\int_{\mathbb{T}^{n}} \bar{H}^{k}(P) d \sigma^{k} \\
& =\int_{\left\{h \geq \bar{H}^{k}-\lambda\right\}} \bar{H}^{k}(P) d \sigma^{k}+\int_{\left\{h<\bar{H}^{k}-\lambda\right\}} \bar{H}^{k}(P) d \sigma^{k} \\
& \leq \int_{\left\{h \geq \bar{H}^{k}-\lambda\right\}} H\left(D u^{k}, x\right)+\lambda d \sigma^{k}+\int_{\left\{h<\bar{H}^{k}-\lambda\right\}} \bar{H}^{k}(P) e^{-k \lambda} d x \\
& \leq \int_{\mathbb{T}^{n}} H\left(D u^{k}, x\right) d \sigma^{k}+\lambda+C e^{-k \lambda},
\end{aligned}
$$

where $h:=H\left(D u^{k}, x\right)$. Thus

$$
\bar{H}(P)=\lim _{k \rightarrow \infty} \bar{H}^{k}(P) \leq \liminf _{k \rightarrow \infty} \int_{\mathbb{T}^{n}} H\left(D u^{k}, x\right) d \sigma^{k}+\lambda,
$$

and, since $\lambda>0$ is arbitrary, (3.12) follows.

3. We employ (3.12) to compute

$$
\begin{aligned}
& \int_{\mathbb{R}^{n}} \int_{\mathbb{T}^{n}} L(q, x) d \mu=\lim _{k \rightarrow \infty} \int_{\mathbb{T}^{n}} L\left(D_{p} H\left(D u^{k}, x\right), x\right) d \sigma^{k} \\
&= \lim _{k \rightarrow \infty} \int_{\mathbb{T}^{n}} D u^{k} \cdot D_{p} H\left(D u^{k}, x\right)-H\left(D u^{k}, x\right) d \sigma^{k} \\
&= \lim _{k \rightarrow \infty}\left\{P \cdot \int_{\mathbb{T}^{n}} D_{p} H\left(D u^{k}, x\right) d \sigma^{k}\right. \\
&\left.\quad+\int_{\mathbb{T}^{n}} D v^{k} \cdot D_{p} H\left(D u^{k}, x\right) d \sigma^{k}-\int_{\mathbb{T}^{n}} H\left(D u^{k}, x\right) d \sigma^{k}\right\} \\
& \leq P \cdot Q-\bar{H}(P) \leq \max _{R}\{R \cdot Q-\bar{H}(R)\}=\bar{L}(Q) .
\end{aligned}
$$

But Mather's minimization principle asserts that

$$
\bar{L}(Q)=\inf \left\{\int_{\mathbb{R}^{n}} \int_{\mathbb{T}^{n}} L(q, x) d \mu \mid \mu \text { is flow invariant, } \int_{\mathbb{R}^{n}} \int_{\mathbb{T}^{n}} q d \mu=Q\right\}
$$

Hence equality holds in each step of the foregoing estimate, and in particular $Q \in \partial \bar{H}(P), P \in$ $\partial \bar{L}(Q)$. Furthermore, the limit (3.7) must hold. 
4. To study the differentiability of $u$, we mimic a technique from [E-G]. Since $H(D u, x) \leq$ $\bar{H}(P)$ a.e. in $\mathbb{T}^{n}$, the mollified function $u^{\varepsilon}:=\eta_{\varepsilon} * u$ satisfies

$$
\beta_{\varepsilon}(x)+H\left(D u^{\varepsilon}, x\right) \leq \bar{H}(P)+O(\varepsilon)
$$

in $\mathbb{T}^{n}$, for

$$
\beta_{\varepsilon}(x):=\frac{\gamma}{2} \int_{\mathbb{R}^{n}} \eta_{\varepsilon}(x-y)\left|D u(y)-D u^{\varepsilon}(x)\right|^{2} d y .
$$

See the proof of Theorem 4.1 in $[\mathrm{E}-\mathrm{G}]$ for more details.

Because $q \mapsto L(q, x)$ is uniformly convex, we have

$$
\frac{\gamma}{2}|q-r|^{2} \leq L(q, x)-L(r, x)-D_{q} L(r, x) \cdot(q-r)
$$

for each $q, r \in \mathbb{R}^{n}, x \in \mathbb{T}^{n}$. We select

$$
r=D_{p} H\left(D u^{\varepsilon}(x), x\right)
$$

and integrate with respect to $\mu$ :

$$
\begin{aligned}
& \frac{\gamma}{2} \int_{\mathbb{R}^{n}} \int_{\mathbb{T}^{n}}\left|q-D_{p} H\left(D u^{\varepsilon}, x\right)\right|^{2} d \mu \\
& \leq \int_{\mathbb{R}^{n}} \int_{\mathbb{T}^{n}} L(q, x)-L\left(D_{p} H\left(D u^{\varepsilon}, x\right), x\right) \\
& \quad-D_{q} L\left(D_{p} H\left(D u^{\varepsilon}, x\right), x\right) \cdot\left(q-D_{p} H\left(D u^{\varepsilon}, x\right)\right) d \mu .
\end{aligned}
$$

Now $D_{q} L\left(D_{p} H(p, x), x\right)=p$ and $L\left(D_{p} H(p, x), x\right)=p \cdot D_{p} H(p, x)-H(p, x)$ for all $p \in \mathbb{R}^{n}$, $x \in \mathbb{T}^{n}$. Substituting above, we find

$$
\begin{aligned}
& \frac{\gamma}{2} \int_{\mathbb{R}^{n}} \int_{\mathbb{T}^{n}}\left|q-D_{p} H\left(D u^{\varepsilon}, x\right)\right|^{2} d \mu \\
& \quad \leq \bar{L}(Q)+\int_{\mathbb{R}^{n}} \int_{\mathbb{T}^{n}} H\left(D u^{\varepsilon}, x\right)-D u^{\varepsilon} \cdot D_{p} H\left(D u^{\varepsilon}, x\right)-D u^{\varepsilon} \cdot\left(q-D p H\left(D u^{\varepsilon}, x\right)\right) d \mu .
\end{aligned}
$$

Hence (3.6) and (3.13) give

$$
\begin{aligned}
\frac{\gamma}{2} \int_{\mathbb{R}^{n}} & \int_{\mathbb{T}^{n}}\left|q-D_{p} H\left(D u^{\varepsilon}, x\right)\right|^{2} d \mu+\int_{\mathbb{T}^{n}} \beta_{\varepsilon} d \sigma \\
& \leq \bar{L}(Q)+\bar{H}(P)-\int_{\mathbb{R}^{n}} \int_{\mathbb{T}^{n}}\left(P+D v^{\varepsilon}\right) \cdot q d \mu+O(\varepsilon) \\
& =\bar{L}(Q)+\bar{H}(P)-P \cdot Q+O(\varepsilon)=O(\varepsilon) .
\end{aligned}
$$

5. We now show that this estimate implies $u$ is differentiable $\sigma$-a.e. (This was easier in the earlier paper $[\mathrm{E}-\mathrm{G}]$ where we dealt with a semiconcave function $u$.) 
The inequality (3.17) implies

$$
\int_{\mathbb{T}^{n}} \beta_{\varepsilon}^{1 / 2} d \sigma \leq\left(\int_{\mathbb{T}^{n}} \beta_{\varepsilon} d \sigma\right)^{1 / 2} \leq C \varepsilon^{1 / 2}
$$

for each $\varepsilon>0$. Let $\varepsilon=1 / 2^{k}$ and sum, to deduce

$$
\int_{\mathbb{T}^{n}} \sum_{k=1}^{\infty} \beta_{1 / 2^{k}}^{1 / 2} d \sigma<\infty
$$

Hence

$$
\sum_{k=1}^{\infty} \beta_{1 / 2^{k}}^{1 / 2}(x)<\infty
$$

for $\sigma$-a.e. point $x \in \mathbb{T}^{n}$. Fix such a point $x$. For some constant $\rho>0$, we have

$$
\beta_{2 r}^{1 / 2}(x) \geq \rho\left(f_{B(x, r)}\left|D u-(D u)_{x, r}\right|^{2} d y\right)^{1 / 2} \geq \rho f_{B(x, r)}\left|D u-(D u)_{x, r}\right| d y,
$$

where

$$
(D u)_{x, r}:=f_{B(x, r)} D u d y
$$

Thus (3.18) implies

$$
\sum_{k=1}^{\infty} f_{B\left(x, 1 / 2^{k}\right)}\left|D u-(D u)_{x, 1 / 2^{k}}\right| d y<\infty .
$$

Since

$$
\left|(D u)_{x, 1 / 2^{k+1}}-(D u)_{x, 1 / 2^{k}}\right| \leq C f_{B\left(x, 1 / 2^{k}\right)}\left|D u-(D u)_{x, 1 / 2^{k}}\right| d y,
$$

the limit $D u(x):=\lim _{k \rightarrow \infty}(D u)_{x, 1 / 2^{k}}$ exists. Next, for each $r>0$ we have the estimate

$$
f_{B(x, r)}|u(y)-u(x)-D u(x) \cdot(y-x)| d y \leq C r f_{B(x, r)}|D u(y)-D u(x)| d y=o(r) .
$$

Since $u$ is Lipschitz continuous, in fact

$$
\max _{y \in B(x, r)}\{|u(y)-u(x)-D u(x) \cdot(y-x)|\}=o(r) \quad \text { as } r \rightarrow 0,
$$

and so $u$ is differentiable at $x$.

6. The foregoing calculations show that

$$
D u^{\varepsilon}(x) \rightarrow D u(x) \quad \text { boundedly, } \sigma \text {-almost everywhere. }
$$


Thus passing to limits in (3.17) gives

$$
\int_{\mathbb{R}^{n}} \int_{\mathbb{T}^{n}}\left|q-D_{p} H(D u, x)\right|^{2} d \mu=0
$$

and (3.9) follows.

7. If $H(D u, x)<\bar{H}(P)$ on a set of positive $\sigma$ measure, we set

$$
r=D_{p} H(D u(x), x)
$$

in the inequality in (3.15) and integrate with respect to $\sigma$. Calculating as in step 4 above, we deduce that

$$
P \cdot Q<\bar{L}(Q)+\bar{H}(P),
$$

a contradiction. Thus (3.10) is valid, as is (3.11) since

$$
0=\int_{\mathbb{R}^{n}} \int_{\mathbb{T}^{n}} q \cdot D \phi d \mu=\int_{\mathbb{T}^{n}} D_{p} H(D u, x) \cdot D \phi d \sigma
$$

for each $\phi \in C^{1}\left(\mathbb{T}^{n}\right)$.

Remarks. I do not see how to use PDE estimates based upon (1.11) to recover Mather's theorem that the gradient $D u$ is Lipschitz continuous on the support of the measure $\sigma$. (However, see the PDE proof in $[\mathrm{E}-\mathrm{G}]$. )

\section{Some formulas involving $\overline{\mathbf{H}}^{\mathrm{k}}$.}

First, we establish a simple monotonicity property.

Theorem 4.1. For each $P \in \mathbb{R}^{n}$, we have

$$
\bar{H}^{k}(P) \leq \bar{H}^{l}(P) \quad \text { if } k \leq l .
$$

Proof. We have

$$
I_{k}\left[v^{k}\right]=\int_{\mathbb{T}^{n}} e^{k H\left(D u^{k}, x\right)} d x \leq \int_{\mathbb{T}^{n}} e^{k H\left(D u^{l}, x\right)} d x \leq\left(\int_{\mathbb{T}^{n}} e^{l H\left(D u^{l}, x\right)} d x\right)^{\frac{k}{l}}=\left(I_{l}\left[v^{l}\right]\right)^{\frac{k}{l}} ;
$$

and so

$$
\left(I_{k}\left[v^{k}\right]\right)^{\frac{1}{k}} \leq\left(I_{l}\left[v^{l}\right]\right)^{\frac{1}{l}} .
$$

Take the log of both sides to derive (4.1).

Since the minimization problem (1.2) corresponds to a nonlinearity which is uniformly convex in the variable $p$, the unique minimizer $v^{k}$ depends smoothly on the parameter $P \in \mathbb{R}^{n}$. Consequently $\bar{H}^{k}$ is a smooth function, and we can compute its first and second derivatives: 
Theorem 4.2. For $k=1, \ldots$ and $P \in \mathbb{R}^{n}$, we have the formulas

$$
D \bar{H}^{k}(P)=\int_{\mathbb{T}^{n}} D_{p} H\left(D u^{k}, x\right) d \sigma^{k}
$$

and

$$
\begin{aligned}
& D^{2} \bar{H}^{k}(P)=k \int_{\mathbb{T}^{n}}\left(D_{p} H\left(D u^{k}, x\right) D_{x P}^{2} u^{k}-D \bar{H}^{k}(P)\right) \\
& \otimes\left(D_{p} H\left(D u^{k}, x\right) D_{x P}^{2} u^{k}-D \bar{H}^{k}(P)\right) d \sigma^{k} \\
&+\int_{\mathbb{T}^{n}} D_{p}^{2} H\left(D u^{k}, x\right) D_{x P}^{2} u^{k}: D_{x P}^{2} u^{k} d \sigma^{k} .
\end{aligned}
$$

In particular, $\bar{H}^{k}$ is a convex function of $P$.

Remark. Our notation means that the $(l, m)^{\text {th }}$ component of the first term on the right hand side of (4.3) is

$$
k \int_{\mathbb{T}^{n}}\left(H_{p_{i}}\left(D u^{k}, x\right) u_{x_{i} P_{l}}^{k}-\bar{H}_{P_{l}}^{k}(P)\right)\left(H_{p_{j}}\left(D u^{k}, x\right) u_{x_{j} P_{m}}^{k}-\bar{H}_{P_{m}}^{k}(P)\right) d \sigma^{k}
$$

and of the second term is

$$
\int_{\mathbb{T}^{n}} H_{p_{i} p_{j}}\left(D u^{k}, x\right) u_{x_{i} P_{l}}^{k} u_{x_{j} P_{m}}^{k} d \sigma^{k} .
$$

Proof. 1. According to (1.9), we have

$$
e^{k \bar{H}^{k}(P)}=\int_{\mathbb{T}^{n}} e^{k H\left(D u^{k}, x\right)} d x .
$$

Differentiate with respect to $P_{l}$ :

$$
\begin{aligned}
k e^{k \bar{H}^{k}} \bar{H}_{P_{l}}^{k} & =\int_{\mathbb{T}^{n}} e^{k H\left(D u^{k}, x\right)} k H_{p_{i}} u_{P_{l} x_{i}}^{k} d x \\
& =\int_{\mathbb{T}^{n}} e^{k H\left(D u^{k}, x\right)} k H_{p_{i}}\left(\delta_{l, i}+v_{P_{l} x_{i}}^{k}\right) d x \\
& =\int_{\mathbb{T}^{n}} e^{k H\left(D u^{k}, x\right)} k H_{p_{l}} d x .
\end{aligned}
$$

The last equality holds since $u^{k}$ solves the Euler-Lagrange equation (1.7). We cancel the $k$, rearrange, and derive formula (4.2).

2. As above, write out the $l^{\text {th }}$ component of (4.2), and differentiate with respect to $P_{m}$ :

$$
\begin{aligned}
e^{k \bar{H}^{k}}\left(\bar{H}_{P_{l} P_{m}}^{k}+k \bar{H}_{P_{l}}^{k} \bar{H}_{P_{m}}^{k}\right) & =\int_{\mathbb{T}^{n}} e^{k H\left(D u^{k}, x\right)}\left(H_{p_{i}} u_{P_{m} P_{l} x_{i}}^{k}+H_{p_{i} p_{j}} u_{P_{l} x_{i}}^{k} u_{P_{m} x_{j}}^{k}\right) d x \\
& +k \int_{\mathbb{T}^{n}} e^{k H\left(D u^{k}, x\right)} H_{p_{i}} u_{P_{l} x_{i}}^{k} H_{p_{j}} u_{P_{m} x_{j}}^{k} d x .
\end{aligned}
$$


The integral of the first term on the right is zero, according to (1.7), and, recalling (4.2), we can recast the remaining expressions into formula (4.3).

Nonresonance and averaging. As an application of these formulas, we recast and simplify some ideas from $\S 9$ of $[\mathrm{E}-\mathrm{G}]$. Assume that $\bar{H}$ is differentiable at $P$ and that $Q=D \bar{H}(P)$ satisfies the nonresonance condition:

$$
Q \cdot m \neq 0 \quad \text { for each vector } m \in \mathbb{Z}^{n}, m \neq 0 \text {. }
$$

Theorem 4.3. Suppose also that $D^{2} \bar{H}^{k}(P)$ is bounded as $k \rightarrow \infty$. Then

$$
\lim _{k \rightarrow \infty} \int_{\mathbb{T}^{n}} \Phi\left(D_{P} u^{k}(P, x)\right) d \sigma^{k}=\int_{\mathbb{T}^{n}} \Phi(X) d X
$$

for each continuous, $\mathbb{T}^{n}$-periodic function $\Phi$.

Proof. Observe first that the function

$$
e^{2 \pi i m \cdot D_{P} u^{k}}=e^{2 \pi i m \cdot x} e^{2 \pi i m \cdot D_{P} v^{k}}
$$

is $\mathbb{T}^{n}$-periodic. Hence we have

$$
\begin{aligned}
0= & \int_{\mathbb{T}^{n}} D_{p} H\left(D u^{k}, x\right) \cdot D_{x}\left(e^{2 \pi i m \cdot D_{P} u^{k}}\right) d \sigma^{k} \\
= & 2 \pi i \int_{\mathbb{T}^{n}} e^{2 \pi i m \cdot D_{P} u^{k}} m \cdot D_{p} H\left(D u^{k}, x\right) D_{x P}^{2} u^{k} d \sigma^{k} \\
= & 2 \pi i \int_{\mathbb{T}^{n}} e^{2 \pi i m \cdot D_{P} u^{k}} m \cdot D_{P} \bar{H}^{k}(P) d \sigma^{k} \\
& \quad+2 \pi i \int_{\mathbb{T}^{n}} e^{2 \pi i m \cdot D_{P} u^{k}} m \cdot\left(D_{p} H\left(D u^{k}, x\right) D_{x P}^{2} u^{k}-D_{P} \bar{H}^{k}(P)\right) d \sigma^{k} .
\end{aligned}
$$

Consequently if $m \neq 0$, the identity (4.3) implies

$$
\begin{aligned}
\left|\left(m \cdot D_{P} \bar{H}^{k}(P)\right) \int_{\mathbb{T}^{n}} e^{2 \pi i m \cdot D_{P} u^{k}} d \sigma^{k}\right| & \left.\leq|m| \int_{\mathbb{T}^{n}} \mid D_{p} H\left(D u^{k}, x\right) D_{x P}^{2} u^{k}-D_{P} \bar{H}^{k}(P)\right) \mid d \sigma^{k} \\
& \leq|m|\left(k^{-1} \Delta \bar{H}^{k}(P)\right)^{\frac{1}{2}} .
\end{aligned}
$$

Since $D \bar{H}^{k}(P) \rightarrow D \bar{H}(P)=Q$ and $m \cdot Q \neq 0$, we deduce that

$$
\lim _{k \rightarrow \infty} \int_{\mathbb{T}^{n}} e^{2 \pi i m \cdot D_{P} u^{k}} d \sigma^{k}=0
$$

and so

$$
\lim _{k \rightarrow \infty} \int_{\mathbb{T}^{n}} \Phi\left(D_{P} u^{k}(P, x)\right) d \sigma^{k}=\int_{\mathbb{T}^{n}} \Phi(X) d X
$$


for each periodic function $\Phi$ whose Fourier expansion contains only finitely many nonzero terms. Such functions are dense in the sup-norm, and so we deduce (4.5).

Remark. As explained in a different setting in [E-G], Theorem 4.3 provides a partial interpretation of the following heuristics.

Suppose that we regard $u=u(P, x)$ as a smooth generating function, inducing the canonical change of variables $(p, x) \rightarrow(P, X)$, where $p=D_{x} u(P, x), X=D_{P} u(P, x)$. Then the corresponding Hamiltonian dynamics become

$$
\left\{\begin{array}{l}
\dot{\mathbf{X}}=D \bar{H}(\mathbf{P}) \\
\dot{\mathbf{P}}=0
\end{array}\right.
$$

and consequently $\mathbf{X}(t)=Q t+X_{0}$. According to the nonresonance condition, we then have

$$
\lim _{T \rightarrow \infty} \frac{1}{T} \int_{0}^{T} \Phi(\mathbf{X}(t)) d t=\int_{\mathbb{T}^{n}} \Phi(X) d X
$$

\section{Appendix A: Uniform estimates on $\mathrm{Du}^{\mathrm{k}}$.}

Theorem 5.1. There exists a constant $C$ such that

$$
\max _{\mathbb{T}^{n}} H\left(D u^{k}, x\right) \leq \bar{H}^{k}(P)+\frac{C \log k}{k}
$$

for $k=2, \ldots$

Remark. Since $H$ grows quadratically in the variable $p$ and $\bar{H}^{k}(P) \leq \bar{H}(P)$, this implies the bound

$$
\max _{\mathbb{T}^{n}}\left|D u^{k}\right| \leq C
$$

for $k=1, \ldots$ Furthermore, the min-max formula (1.1) implies $\bar{H}(P) \leq \max _{x \in \mathbb{T}^{n}} H\left(D u^{k}, x\right)$; and hence Theorems 4.1 and 5.1 provide the two-sided bounds

$$
\bar{H}^{k}(P) \leq \bar{H}(P) \leq \bar{H}^{k}(P)+\frac{C \log k}{k}
$$

for $k=2, \ldots$ The constant $C$ is bounded for $P$ in bounded subsets of $\mathbb{R}^{n}$. 
Proof. 1. To ease notation, we drop the superscripts $k$. Multiply the Euler-Lagrange equation $\operatorname{div}\left(\sigma D_{p} H\right)=0$ by $\operatorname{div}\left(\sigma^{p} D_{p} H\right)$ for $p \geq 0$, and integrate by parts:

$$
\begin{aligned}
0 & =\int_{\mathbb{T}^{n}}\left(\sigma H_{p_{i}}\right)_{x_{j}}\left(\sigma^{p} H_{p_{j}}\right)_{x_{i}} d x \\
& =\int_{\mathbb{T}^{n}}\left(\sigma\left(H_{p_{i}}\right)_{x_{j}}+\sigma_{x_{j}} H_{p_{i}}\right)\left(\sigma^{p}\left(H_{p_{j}}\right)_{x_{i}}+p \sigma^{p-1} \sigma_{x_{i}} H_{p_{j}}\right) d x \\
& =\int_{\mathbb{T}^{n}} \sigma^{p+1}\left(H_{p_{i}}\right)_{x_{j}}\left(H_{p_{j}}\right)_{x_{i}}+p \sigma^{p-1} H_{p_{i}} \sigma_{x_{i}} H_{p_{j}} \sigma_{x_{j}}+(p+1) \sigma^{p} \sigma_{x_{i}} H_{p_{j}}\left(H_{p_{i}}\right)_{x_{j}} d x \\
& =: \int_{\mathbb{T}^{n}} A+B+C d x .
\end{aligned}
$$

Recalling our hypotheses on $H$, we see that

$$
\begin{aligned}
A & =\sigma^{p+1}\left(H_{p_{i} p_{m}} u_{x_{m} x_{j}}+H_{p_{i} x_{j}}\right)\left(H_{p_{j} p_{l}} u_{x_{l} x_{i}}+H_{p_{j} x_{i}}\right) \\
& \geq \sigma^{p+1}\left(\gamma^{2}\left|D^{2} u\right|^{2}-C(1+|D u|)\left|D^{2} u\right|-C\left(1+|D u|^{2}\right)\right) \\
& \geq-C \sigma^{p+1}\left(1+|D u|^{2}\right) .
\end{aligned}
$$

Clearly $B \geq 0$, and we further calculate that

$$
\begin{aligned}
C & =(p+1) \sigma^{p} \sigma_{x_{i}} H_{p_{j}}\left(H_{p_{i} p_{l}} u_{x_{l} x_{j}}+H_{p_{i} x_{j}}\right) \\
& =\frac{(p+1)}{k} \sigma^{p-1} H_{p_{i} p_{l}} \sigma_{x_{i}} \sigma_{x_{l}}+(p+1) \sigma^{p} \sigma_{x_{i}}\left(H_{p_{j}} H_{p_{i} x_{j}}-H_{p_{i} p_{l}} H_{x_{l}}\right), \\
& \geq \frac{(p+1)}{k} \gamma \sigma^{p-1}|D \sigma|^{2}-C(p+1) \sigma^{p}|D \sigma|\left(1+|D u|^{2}\right),
\end{aligned}
$$

since $\sigma_{x_{l}}=\sigma k h_{x_{l}}=\sigma k\left(H_{p_{j}} u_{x_{l} x_{j}}+H_{x_{l}}\right)$.

2. We combine the foregoing estimates, to compute

$$
\begin{aligned}
\frac{p+1}{k} \int_{\mathbb{T}^{n}} \sigma^{p-1}|D \sigma|^{2} d x \leq C & \int_{\mathbb{T}^{n}} \sigma^{p+1}\left(1+|D u|^{2}\right) d x \\
& +C(p+1) \int_{\mathbb{T}^{n}} \sigma^{p}|D \sigma|\left(1+|D u|^{2}\right) d x .
\end{aligned}
$$


Hence

$$
\int_{\mathbb{T}^{n}} \sigma^{p-1}|D \sigma|^{2} d x \leq C k^{2} \int_{\mathbb{T}^{n}} \sigma^{p+1}\left(1+|D u|^{4}\right) d x .
$$

Sobolev's inequality provides the estimate

$$
\begin{aligned}
\left(\int_{\mathbb{T}^{n}} \sigma^{(p+1)(1+\theta)} d x\right)^{\frac{1}{1+\theta}} & \leq C \int_{\mathbb{T}^{n}}\left|D \sigma^{\frac{p+1}{2}}\right|^{2}+\sigma^{p+1} d x \\
& \leq C(p+1)^{2} \int_{\mathbb{T}^{n}} \sigma^{p-1}|D \sigma|^{2} d x+C \int_{\mathbb{T}^{n}} \sigma^{p+1} d x
\end{aligned}
$$

where $\theta>0$ is the same as in the proof of Lemma 3.1.

Therefore (5.5) implies

$$
\left(\int_{\mathbb{T}^{n}} \sigma^{(p+1)(1+\theta)} d x\right)^{\frac{1}{1+\theta}} \leq C(p+1)^{2} k^{2} \int_{\mathbb{T}^{n}} \sigma^{p+1}\left(1+|D u|^{4}\right) d x .
$$

Write $\beta:=(1+\theta)^{1 / 2}>1$ and calculate

$$
|D u|^{4} \leq C\left(1+h^{2}\right) \leq C\left(1+(h-\bar{H})_{+}^{2}\right) \leq C\left(1+\sigma^{\frac{\beta-1}{\beta}}\right) .
$$

Consequently,

$$
\left(\int_{\mathbb{T}^{n}} \sigma^{(p+1) \beta^{2}} d x\right)^{\frac{1}{\beta^{2}}} \leq C(p+1)^{2} k^{2} \int_{\mathbb{T}^{n}} \sigma^{p+1}\left(1+\sigma^{\frac{\beta-1}{\beta}}\right) d x .
$$

Since

$$
\int_{\mathbb{T}^{n}} \sigma^{p+1} \sigma^{\frac{\beta-1}{\beta}} d x \leq\left(\int_{\mathbb{T}^{n}} \sigma^{(p+1) \beta} d x\right)^{1 / \beta}\left(\int_{\mathbb{T}^{n}} \sigma d x\right)^{1-\frac{1}{\beta}}=\left(\int_{\mathbb{T}^{n}} \sigma^{(p+1) \beta} d x\right)^{1 / \beta},
$$

we deduce

$$
\left(\int_{\mathbb{T}^{n}} \sigma^{(p+1) \beta^{2}} d x\right)^{1 / \beta^{2}} \leq C(p+1)^{2} k^{2}\left(\int_{\mathbb{T}^{n}} \sigma^{(p+1) \beta} d x\right)^{1 / \beta}
$$

that is,

$$
\left(\int_{\mathbb{T}^{n}} \sigma^{q \beta} d x\right)^{1 / \beta} \leq C q^{2 \beta} k^{2 \beta} \int_{\mathbb{T}^{n}} \sigma^{q} d x
$$

for each $q=(p+1) \beta \geq \beta$. 
3. We next employ Moser's method of estimating higher and higher $L^{p}$ norms. For this, set $q=q_{m}:=\beta^{m}$ in (5.7):

$$
\left(\int_{\mathbb{T}^{n}} \sigma^{q_{m+1}} d x\right)^{1 / q_{m+1}} \leq C^{\frac{1}{q_{m}}} q_{m}^{\frac{2 \beta}{q_{m}}} k^{\frac{2 \beta}{q_{m}}}\left(\int_{\mathbb{T}^{n}} \sigma^{q_{m}} d x\right)^{1 / q_{m}},
$$

for $m=1, \ldots$ Iterating, we find

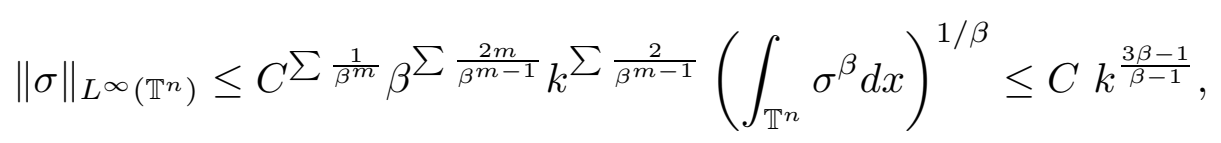

where we used (3.4) to estimate the next-to-last term. But since $\sigma=e^{k(h-\bar{H})}$, this implies

$$
k(h-\bar{H})=\log \sigma \leq \log C+\frac{3 \beta-1}{\beta-1} \log k,
$$

and so

$$
h \leq \bar{H}+\frac{C \log k}{k}
$$

for another constant $C$.

Solving the Euler-Lagrange equations. As an application, we discuss finding smooth solutions of the Euler-Lagrange PDE (1.7).

Theorem 5.2. For each $k=1, \ldots$, there exists a unique, smooth solution $u^{k}=P \cdot x+v^{k}$ of the Euler-Lagrange equation (1.7), where $v^{k}$ is $\mathbb{T}^{n}$-periodic and $\int_{\mathbb{T}^{n}} v^{k} d x=0$.

Proof. 1. Define for each $0 \leq \lambda \leq 1$ the Hamiltonian

$$
H_{\lambda}(p, x):=\lambda H(p, x)+(1-\lambda) \frac{|p|^{2}}{2},
$$

for $p, x \in \mathbb{R}^{n}$; and for each $\mathrm{k}$ introduce the PDE

$$
\operatorname{div}\left(e^{k H_{\lambda}(D u, x)} D_{p} H_{\lambda}(D u, x)\right)=0
$$

where $u=P \cdot x+v, v$ being $\mathbb{T}^{n}$-periodic with $\int_{\mathbb{T}^{n}} v d x=0$.

Define

$$
\Lambda:=\left\{\lambda \mid 0 \leq \lambda \leq 1,\left(5.8_{\lambda}\right) \text { has a smooth solution }\right\} .
$$

Clearly we have $0 \in \Lambda$, corresponding to the solution $u=P \cdot x$; that is, $v \equiv 0$.

2. We assert next that $\Lambda$ is closed. This is a consequence of Theorem 5.1, since the Hamiltonians $H_{\lambda}$ each satisfy the basic assumptions. Consequently, we can bound the first derivatives of solutions of $\left(5.8_{\lambda}\right)$, and elliptic regularity theory implies we can then estimate their derivatives of any order. These bounds are independent of $\lambda \in \Lambda$. 
So any convergent subsequence of elements of $\Lambda$ contains a further subsequence whose corresponding solutions of the PDE converge uniformly, along with all derivatives.

3. Finally, we claim that $\Lambda$ is open. To see this, take any $\lambda \in \Lambda$ and write out the linearization of $\left(5.8_{\lambda}\right)$ about the smooth solution $u=P \cdot x+v$ :

$$
L w:=-\left(e^{k H_{\lambda}(D u, x)}\left(H_{\lambda, p_{i} p_{j}}(D u, x)+k H_{\lambda, p_{i}}(D u, x) H_{\lambda, p_{j}}(D u, x)\right) w_{x_{j}}\right)_{x_{i}} .
$$

This is a symmetric, uniformly elliptic operator, whose null space among periodic functions consists of the constants. Using the Implicit Function Theorem, we construct a unique solution for nearby values of $\lambda$.

4. Since $\Lambda$ is nonempty, closed and open, we have $\Lambda=[0,1]$ and in particular $1 \in \Lambda$. Thus the Euler-Lagrange PDE has a smooth solution $u=P \cdot x+v$. It is unique since any such solution gives the unique minimizer of $I_{k}[\cdot]$, subject to the requirement that $\int_{\mathbb{T}^{n}} v d x=0$.

\section{Appendix B: A quick proof of the min-max formula.}

The following argument is due to A. Fathi.

Let $u$ be a viscosity solution of $H(D u, x)=\bar{H}(P)$ and let $\sigma$ be a corresponding Mather measure, so that $\operatorname{div}\left(\sigma D_{p} H(D u, x)\right)=0$. Take $\hat{u}=P \cdot x+\hat{v}$, where $\hat{v}$ any $\mathbb{T}^{n}$-periodic, $C^{1}$ function. Then convexity implies

$$
H(D u, x)+D_{p} H(D u, x) \cdot(D \hat{u}-D u) \leq H(D \hat{u}, x) .
$$

We integrate with respect to $\sigma$ and deduce thereby that

$$
\bar{H}(P)=\int_{\mathbb{T}^{n}} H(D u, x) d \sigma \leq \int_{\mathbb{T}^{n}} H(D \hat{u}, x) d \sigma \leq \max _{x \in \mathbb{T}^{n}} H(D \hat{u}, x) .
$$

Thus $\bar{H}(P) \leq \inf _{\hat{v} \in C^{1}} \max _{x \in \mathbb{T}^{n}} H(P+D \hat{v}, x)$.

On the other hand, if $\eta_{\delta}$ is a radial convolution kernel, the smoothed function $u_{\delta}:=$ $\eta_{\delta} * u=P \cdot x+v_{\delta}$ satisfies

$$
H\left(D u_{\delta}, x\right) \leq \bar{H}(P)+O(\delta)
$$

on $\mathbb{T}^{n}$. Therefore $\inf _{\hat{v} \in C^{1}} \max _{x \in \mathbb{T}^{n}} H(P+D \hat{v}, x) \leq \bar{H}(P)$. 


\section{REFERENCES}

[A-1] G. Aronsson, Minimization problems for the functional sup $F\left(x, f(x), f^{\prime}(x)\right)$, Ark. Mat. 6 (1965), $33-53$.

[A-2] G. Aronsson, Extension of functions satisfying Lipschitz conditions, Ark. Mat. 6 (1967), 551-561.

[B] E. N. Barron, Viscosity solutions and analysis in $L^{\infty}$, in Nonlinear Analysis, Differential Equations and Control (1999), Dordrecht, 1-60.

[B-J-W] E. N. Barron, R. Jensen and C. Y. Wang, The Euler equation and absolute minimizers of $L^{\infty}$ functionals, Arch. Ration. Mech. Analysis 157 (2001), 255-283.

[C-I-P] G. Contreras, R. Iturriaga and G. P. Paternain, Lagrangian graphs, minimizing measures and Mane's critical values, Geom. Funct. Analysis 8 (1998), 788-809.

[D-E] D. M. Duc and J. Eells, Regularity of exponentially harmonic functions, Internat. J. Math 2 (1991), 395-408.

[E-G] L. C. Evans and D. Gomes, Effective Hamiltonians and averaging for Hamiltonian dynamics I, Archive Rational Mech and Analysis 157 (2001), 1-33.

[F1] A. Fathi, Théorème KAM faible et théorie de Mather sur les systèmes lagrangiens, C. R. Acad. Sci. Paris Sr. I Math. 324 (1997), 1043-1046.

[F2] A. Fathi, Solutions KAM faibles conjuguées et barrières de Peierls, C. R. Acad. Sci. Paris Sr. I Math. 325 (1997), 649-652.

[F3] A. Fathi, Weak KAM theory in Lagrangian Dynamics, Preliminary Version, Lecture notes (2001).

[L] G. Lieberman, On the regularity of the minimizer of a functional with exponential growth, Comm. Math. Univ. Carol. 33 (1992), 45-49.

[L-P-V] P.-L. Lions, G. Papanicolaou, and S. R. S. Varadhan, Homogenization of Hamilton-Jacobi equations, unpublished, circa 1988.

[Mt1] J. Mather, Minimal measures, Comment. Math Helvetici 64 (1989), 375-394.

[Mt2] J. Mather, Action minimizing invariant measures for positive definite Lagrangian systems, Math. Zeitschrift 207 (1991), 169-207.

[M-F] J. Mather and G. Forni, Action minimizing orbits in Hamiltonian systems, Transition to Chaos in Classical and Quantum Mechanics, Lecture Notes in Math 1589 (S. Graffi, ed.), Sringer, 1994.

[N] H. Naito, On local Hölder continuity for a minimizer of the exponential energy functional, Nagoya Math. J. 129 (1993), 97-113.

[P] G. P. Paternain, Schrödinger operators with magnetic fields and minimal action functionals, to appear in Israel Journal of Math.

Department of Mathematics, University of California, Berkeley, CA 94720 